\title{
Variantes histológicas del carcinoma urotelial con implicaciones diagnósticas, pronósticas y terapéuticas
}

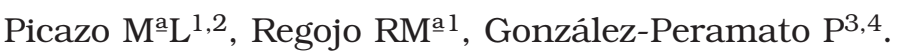 \\ ${ }^{1}$ Departamento de Anatomía Patológica. Hospital Universitario La Paz. Madrid. \\ ${ }^{2}$ Departamento de Anatomía, Histología y Neurociencias. Facultad de Medicina. Universidad Autónoma de \\ Madrid. ${ }^{3}$ Servicio de Anatomía Patológica. Hospital Universitario de Guadalajara. \\ ${ }^{4}$ Departamento de Especialidades Médicas Universidad de Alcalá.
}

Actas Urol Esp. 2007;31(9):989-1001

\section{RESUMEN}

VARIANTES HISTOLÓGICAS DEL CARCINOMA UROTELIAL CON IMPLICACIONES DIAGNÓSTICAS, PRONÓSTICAS Y TERAPÉUTICAS

La mayoría de los carcinomas uroteliales (CU) tienen un patrón de crecimiento histológico de tipo papilar o de tipo sólido; sin embargo, en algunos casos existen variedades histológicas que difieren significativamente de estos patrones habituales En este artículo se han seleccionado aquellas variantes de CU que por su dificultad diagnóstica e implicaciones pronósticas o terapéuticas tienen que ser perfectamente identificadas y conocidas por los patólogos y los urólogos. Las variantes que hemos considerado de mayor interés tanto clínico como patológico han sido: CU variedad en nidos y/o tubular, CU variedad microquística, CU variedad micropapilar, CU con intenso estroma linfoide, tipo linfoepitelioma, CU variedad plasmocitoide y CU sarcomatoide. De cada uno de estos patrones se ha realizado una revisión de la literatura valorando los criterios diagnósticos, el comportamiento clínico y las posibilidades terapéuticas actuales. Además, sugerimos que estas variedades de CU deben ser reflejadas explícitamente en el informe del diagnóstico anatomopatológico, debido a sus implicaciones clínicas.

Palabras clave: Carcinoma de vejiga. Carcinoma urotelial. Tumores vesicales.

\begin{abstract}
HISTOLOGICAL VARIANTS OF UROTHELIAL CARCINOMA WITH DIAGNOSTIC, PROGNOSTIC AND THERAPEUTICAL IMPLICATIONS

Most of urothelial carcinomas (UC) have a pattern of histological growth of papillary or solid type; nevertheless, in some cases there are histological types that significantly differ from these habitual patterns In this paper we have selected those UC variants that by its diagnosis difficulty and therapeutical or prognosis implications have to be perfectly identified and known by pathologists and urologist. The variants that we have considered of greater clinical and pathological interest have been: tubular and/or nested UC, microcystic UC, micropapillary UC, lymphoepithelioma like UC, plasmacytoid UC and sarcomatoid UC. A revision of the literature has been made of each one of these patterns evaluating the criteria diagnoses, clinical behavior and the present therapeutic options. In addition, we suggest that these UC variants must be explicitly reflected in the pathology report, due to its clinical implications.
\end{abstract}

Keywords: Bladder carcinoma. Urothelial carcinoma. Carcinoma transitional cell. 
A proximadamente el 95\% de los tumores de la vejiga son tumores epiteliales, la mayoría carcinomas transicionales con patrón papilar o sólido que no plantean problemas diagnósticos para el patólogo. Además, es ampliamente conocido, que en los carcinomas transicionales de alto grado, hay áreas focales de diferenciación escamosa o glandular ${ }^{1}$, lo que ha llevado a algunos autores a utilizar el término de carcinoma urotelial (CU) con "diferenciación epitelial mixta" para describir aquellos casos en los hay áreas extensas con dos o más patrones histológicos ${ }^{2}$.

La mejor especialización en uropatología, unida a una mayor experiencia, ha permitido reconocer en las últimas décadas, ciertos patrones histológicos inusuales dentro del CU, como son CU variedad en nidos y/o tubular, CU microquístico, CU micropapilar, CU plasmocitoide, CU con estroma linfoide y CU sarcomatoide. La identificación por el patólogo de estas variantes de CU es importante porque:1) algunas variantes histológicas pueden plantear problemas de diagnóstico diferencial con lesiones epiteliales benignas del urotelio -CU variedad "en nidos y/o tubular", CU "microquístico"-, o con tumores metastáticos CU plasmocitoide-; 2) algunos tipos histológicos están asociados con un pronóstico diferente al del CU convencional -CU "micropapilar", CU sarcomatoide- y 3) el reconocimiento de algunos patrones pueden tener implicaciones terapéuticas -CU tipo linfoepitelioma-.

En la literatura se refieren otras variantes de CU: CU con células gigantes tipo osteoclasto, CU con diferenciación trofoblástica, variante de células claras y carcinoma neuroendocrino de células grandes $^{3}$, que por su carácter excepcionalmente infrecuente no se han incluido en esta revisión.

En general, estos diferentes patrones histológicos suelen estar asociados con áreas más o menos extensas de CU convencional, y deben de ser identificados y reseñados en los informes patológicos, dado que pueden estar presentes e incluso predominar en futuras recidivas y metástasis.

\section{CARCINOMA UROTELIAL VARIEDAD “EN NIDOS" Y/O "TUBULAR"}

En 1979 Stern $^{4}$ publicó el caso de un tumor vesical constituido por una proliferación de nidos uroteliales localizados en la lámina propia y que, a pesar de su recidiva, el autor lo consideró de naturaleza benigna. En 1989, Talbert y Young 5 describieron tres casos de carcinomas uroteliales, que formaban nidos y túbulos de apariencia histológica benigna, pero infiltrantes y muy agresivos. Finalmente en 1992, Murphy y Deana ${ }^{6}$ utilizaron el término "variedad en nidos" del CU para describir esta entidad. Desde entonces se han publicado aproximadamente $80 \operatorname{casos}^{4-19} \mathrm{y}$ su incidencia se estima en un $0,3 \%$ de todos los carcinomas transicionales invasivos ${ }^{12}$. Al igual que en el CU convencional, los pacientes son generalmente varones, mayores de 60 años y el sintoma predominante es la hematuria ${ }^{7,8,12,16,17}$. Esta variedad histológica de CU se caracteriza por la presencia de nidos irregulares (Fig. 1a), de células transicionales, de citoplasma amplio eosinófilo o claro y con escasa atipia nuclear, que infiltran la lámina propia y/o la muscular propia de la vejiga (Fig. 1b); la atipia nuclear es mayor en los nidos que infiltran en profundidad ${ }^{5,7,8,12,16}$. En ocasiones, los nidos se anastomosan formando túmulos cordones o trabéculas (Fig. 2) que en ocasiones son semejantes al papiloma inverti$\mathrm{do}^{5,8,14}$. A veces los nidos y/o túbulos se dilatan $\mathrm{y}$ forman grandes quistes de epitelio simple con secreción eosinofílica en su interior ${ }^{4-8,12,15,17}$. Los nidos están rodeados por un estroma que varía de denso y colagenizado a laxo y mixoide, incluso edematoso o desmoplásico ${ }^{8,15,17}$. El urotelio superficial suele ser normal o presenta ligera displasia $^{3,7,8,16}$, por lo que la citología de orina con frecuencia es negativa. Estos tumores no suelen crecer como masas intravesicales, y, por ello, no se identifican mediante cistoscopia. A pesar de su apariencia citológica benigna, el CU variedad en nidos suele ser en el momento del diagnóstico muy agresivo e infiltrante y se comporta como una neoplasia de alto grado, siendo frecuentes las metástasis ${ }^{5-7,12,14,16}$.

El diagnóstico de la variedad en nidos y/o tubular del CU es de singular importancia, ya que, cuando la biopsia es superficial, puede ser difícil de diferenciar de lesiones benignas del urotelio, entre las que se encuentran la hiperplasia de nidos de von Brunn, la cistitis quística y glandular, el papiloma invertido, el adenoma nefrogénico y el paraganglioma 5,7,8,12. El diagnóstico de 


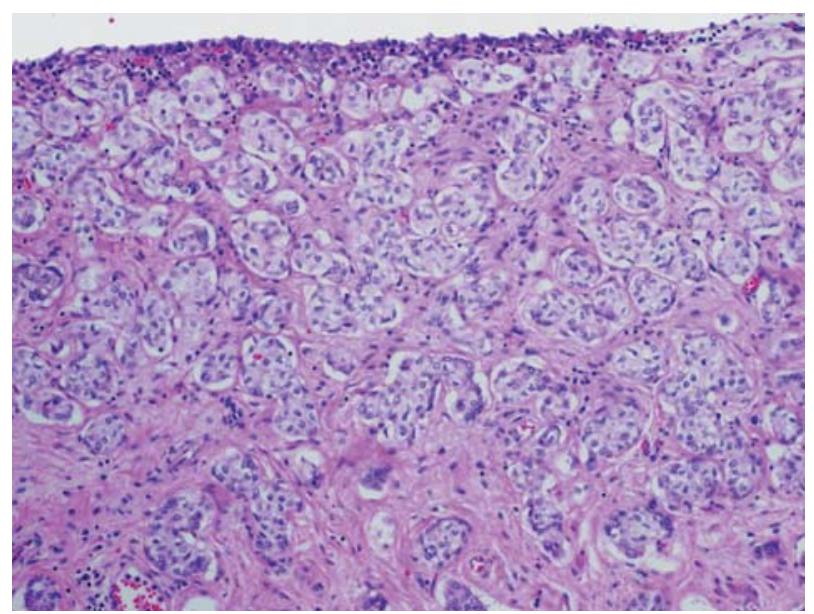

FIGURA 1a. Variedad en nidos de carcinoma urotelial con nidos pequeños, irregulare sin atipia citológica.

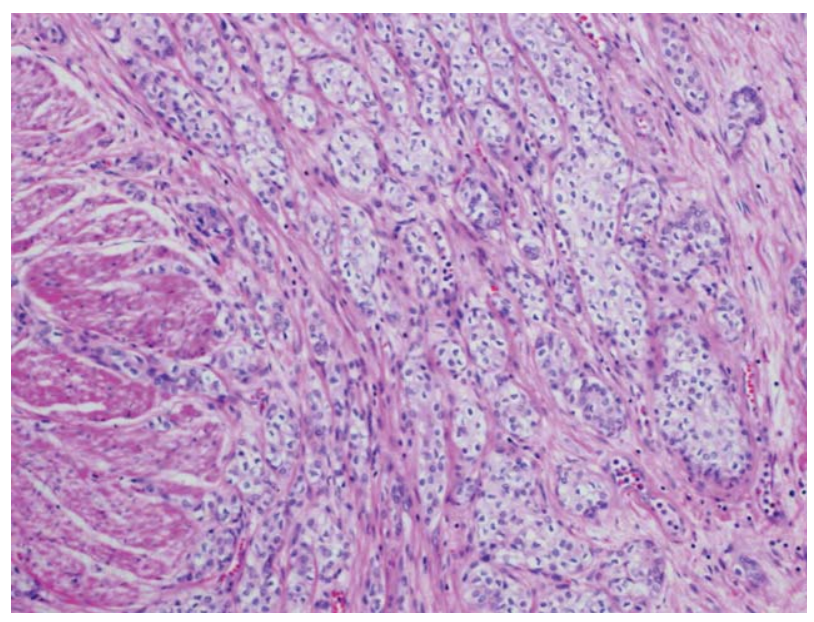

FIGURA $1 b$. Variedad en nidos de carcinoma urotelial que infiltra la muscular propia de la vejiga.

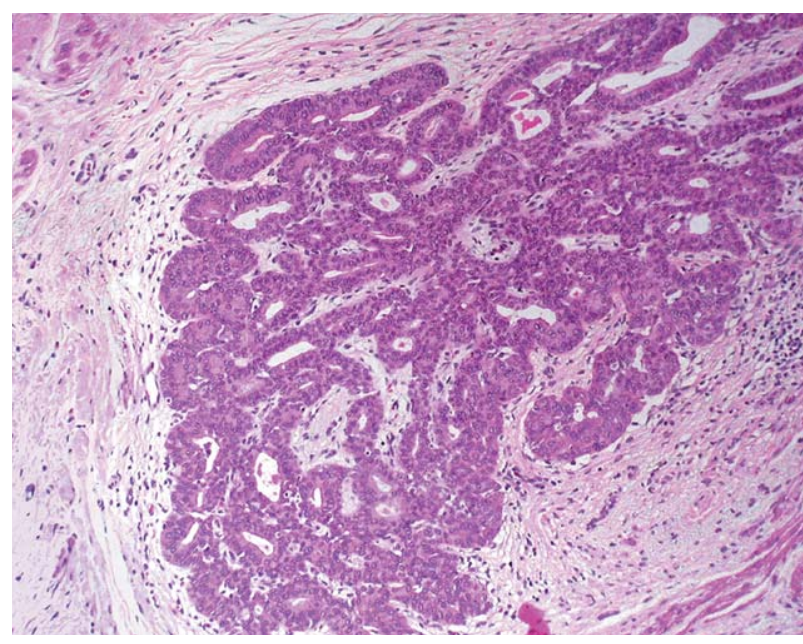

FIGURA 2. Variedad tubular de carcinoma urotelial infiltrando la muscular de la vejiga.
CU variedad en nidos se fundamenta en la presencia de nidos irregulares, de tamaños y formas variables, desordenados y empaquetados, con escaso estroma entre ellos. Además, es clave en el diagnóstico y descarta cualquier entidad benigna, la infiltración de la muscular propia por nidos de células con mayor atipia nuclear $5,7,8,13,16,17$.

Las células tumorales de la variedad en nidos y/o tubular del CU muestran inmunohistoquímicamente positividad intensa y difusa con las citoqueratinas $\mathrm{AE} 1 / \mathrm{AE} 3$ y CK7, y positividad sólo focal con CK20. A pesar de su apariencia histológica benigna, la variedad en nidos del CU comparte algunas características con el CU convencional de alto grado. En este sentido se ha observado, que las células tumorales de los nidos muestran pérdida de expresión de p27 y un elevado índice de proliferación en el componente profundo; sin embargo, otros marcadores biológicos asociados a mal pronóstico en el CU convencional -p53, bc12 y EGF-r- no se expresan en esta variante ${ }^{16}$.

El diagnóstico de $\mathrm{CU}$ variedad en nidos, en biopsias que no incluyen muscular propia, obliga a un seguimiento clínico exhaustivo del paciente y/o a una reevaluación quirúrgica, para descartar infiltración. La agresividad intrínseca de esta variedad histológica y el frecuente retraso en su diagnóstico podrían ser la causa de que estos tumores debuten habitualmente en estadios localmente avanzados. De otra parte, no existen protocolos terapéuticos específicos para los pacientes con esta variedad histológica de CU, debido al escaso número de casos publicados y a la falta de estudios randomizados. Se ha demostrado la ineficacia de la radioterapia y se ha propuesto combinar la cistectomía y la quimiotera$\mathrm{pia}^{12}$. Algunos autores consideran que se debe valorar la realización de cistectomía temprana incluso en estadios pTl ya que, tras la RTU, el seguimiento con citología y cistoscopia es poco eficaz $^{16}$.

\section{CARCINOMA UROTELIAL VARIEDAD "MICROQUÍSTICA"}

La variedad microquística del CU (CUM) se caracteriza por la presencia de numerosos quistes, a veces muy dilatados, generalmente asociados con áreas de CU convencional infiltrante. 
Esta variedad puede presentar, asimismo diferenciación escamosa o diferenciación mucinosa focal $^{20-22}$, aunque en algunos casos el tumor puede ser totalmente quístico ${ }^{20,21}$. Los quistes pueden ser redondos, ovales y a veces elongados y con ramificaciones y pueden alcanzar 1-2 mm de diámetro. Los quistes están generalmente revestidos por una o varias capas de células transicionales o células cuboideas y algunas células pueden tener amplios citoplasmas eosinófilos ${ }^{21}$. En los quistes grandes, el epitelio puede estar aplanado e incluso denudado; frecuentemente su luz contiene material necrótico (Fig. 3a) y ocasionalmente importantes depósitos de calcio ${ }^{20}$. El urotelio que reviste los quistes a veces está perforado por luces pequeñas en las que puede haber una secreción en diana, positiva con las tinciones para mucinas (Fig. 3b). Las estructuras quísticas, variables en morfología y tamaño, se distribuyen irregularmente y tanto en la vejiga como en el urotelio superior ${ }^{21}$, se han descrito infiltrando la pared muscular. Entre los quistes, el estroma puede ser escaso, extremadamente celular o desmoplásico, con importante respuesta inflamatoria crónica. Este estroma es el que permite diferenciar el $\mathrm{CU}$ variedad microquística, de los espacios intercelulares pseudoglandulares que con frecuencia se ven en el seno de los $\mathrm{CU}^{20}$. En $1997 \mathrm{Paz}$ et al. ${ }^{23}$, publicaron una serie de 12 casos de carcinomas uroteliales con microquístes; sin embargo, en nuestra opinión, las imágenes histológicas publicadas parecen corresponder a carcinomas papilares uroteliales con espacios pseudoglandulares y no a la variedad microquística del $\mathrm{CU}$, descrita por Young y Zukerberg ${ }^{20}$.

El CU variedad microquística, puede plantear problemas de diagnóstico diferencial con lesiones quísticas benignas del urotelio (cistitis quística, cistitis glandular y el adenoma nefrogénico) cuando las células tumorales que revisten los quistes tienen moderada o ligera atipia. Este problema se plantea sobre todo en los casos en los que la biopsia es superficial. La varibilidad en el tamaño y contorno de los quistes, su irregular distribución y la infiltración de la pared muscular, son la clave para un correcto diagnostico del CU variedad microquística . El CUM también puede plantear problemas de diagnóstico diferencial con el adenocarcinoma de vejiga. En el ade-

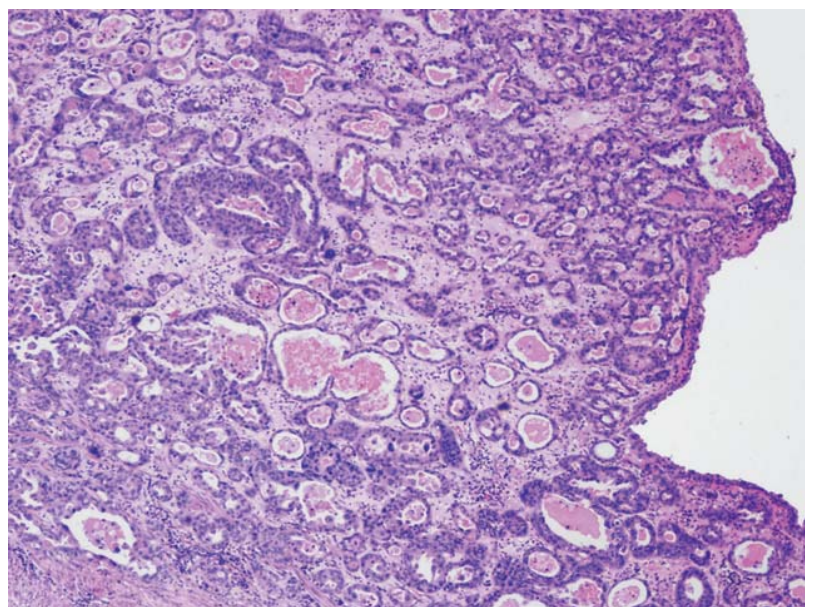

FIGURA 3a. Variedad microquistica de carcinoma urotelial con quistes de diferentes tamaños que crecen irregularmente en la lámina propia.

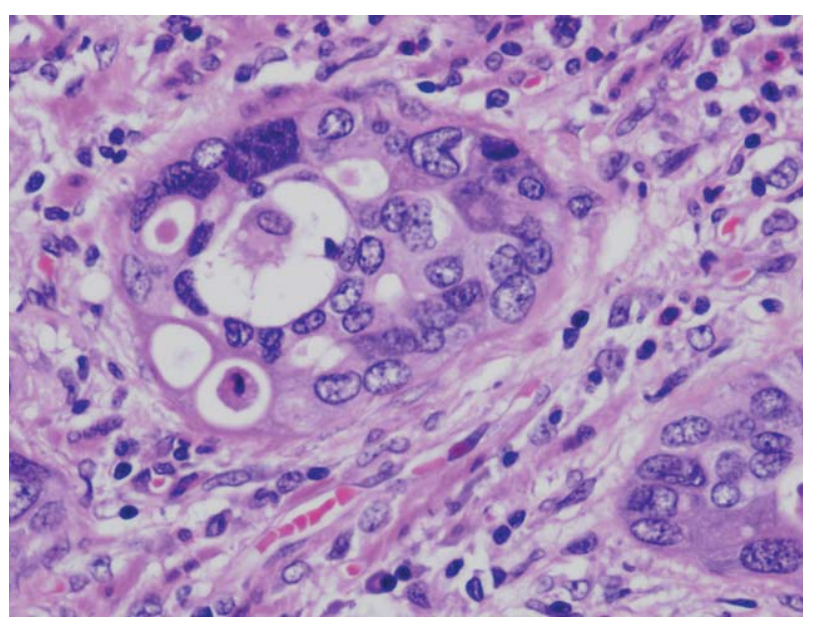

FIGURA 3b. Pequeño quiste con diferenciación de luces con secreción en diana en el revestimiento urotelial.

nocarcinoma, las células tumorales muestran difusamente una diferenciación colónica, mientras que en la variedad microquística del CU, las células que tapizan los quistes son células transicionales y no glandulares, a pesar de la presencia ocasional de células mucinosas en la pared de algunos quistes ${ }^{20}$.

Hay pocos casos descritos de CUM, pero no hay datos que sugiera un pronóstico diferente con respecto a los CUs. convencionales de igual grado y estadio. Sin embargo, se ha sugerido que este patrón debe de constar en los informes patológicos, ya que puede ser de gran ayuda para establecer el origen del tumor primario en casos 
de metástasis; de hecho, en uno de los casos de CU variedad microquística estudiado por nosotros el componente microquístico era focal en la vejiga, pero era el patrón predominante en las metástasis ganglionares (Fig. 4).

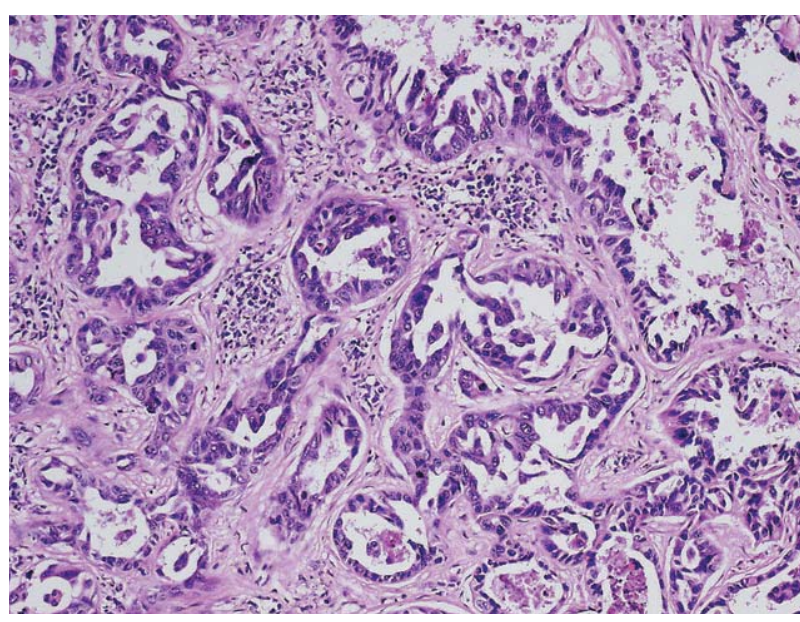

FIGURA 4. Metástasis ganglionar de carcinoma micro quistico con quistes grandes, irregulares y ramificados.

Las variedades en nidos, tubular y microquística del carcinoma transicional comparten algunas similitudes morfológicas, y además, con frecuencia los tres patrones están entremezclados en algunas biopsias. Posiblemente estas variedades representan un espectro histológico que abarca desde los nidos pequeños a los quistes grandes. La ausencia de atipia significativa en las células tumorales plantea los mismos problemas de diagnóstico diferencial con lesiones benignas del urotelio. El patrón arquitectural irregular de los nidos, túbulos y quistes, así como la infiltración de la pared muscular son los criterios básicos para establecer la naturaleza maligna de estas variedades de $\mathrm{CU}$.

\section{CARCINOMA UROTELIAL VARIEDAD “MICROPAPILAR"}

La morfología micropapilar de un carcinoma se ha reconocido como un patrón histológico de valor pronostico en varios órganos, incluidos mama, ovario, pulmón, endometrio, tiroides y también en la vejiga ${ }^{24-28}$. En la vejiga, el CU micropapilar fue descrito por primera vez por Amin en $1994^{26}$ como una variante de CU morfológicamente similar al carcinoma seroso papilar de ovario. Después de la descripción inicial de
Amin, cuya serie incluía 18 casos, se han publicado 6 series más ${ }^{27-32}$ y 9 casos aislados ${ }^{33-41}$, sumando un total de 157 casos, todos localizados en vejiga, excepto tres que se localizaban en el uréter. Con todo, la variante micropapilar de $\mathrm{CU}$ es poco frecuente, con una incidencia entre el $0,2 \%^{29}$ y $6 \%{ }^{31}$ de todos los tipos de CU; su edad de presentación más frecuente varía entre 65 y 69 años. El CU variedad micropapilar es más frecuente en el varón, con una relación varón/mujer variable de $2,3 / 1^{27}$ a $37 / 1^{31}$. Clínicamente no se describen datos diferenciales con respecto a otros $\mathrm{CUs}^{31}$.

La variante micropapilar de CU presenta un patrón histológico bifásico; esto es, es diferente en el componente superficial -pequeñas estructuras filiformes con o sin eje vascular central- que en el componente infiltrante y en las metástasis, caracterizado por grupos redondeados de células muy cohesivas que con frecuencia están rodeados de espacios vacíos (artefactos que simula espacios vasculares pero sin revestimiento de células endoteliales) ${ }^{26,27-32}$. En un número apreciable de casos, se observan trombosis vasculares tumorales verdaderas ${ }^{27}$ (Fig. 5a y b).

El conocimiento de esta variedad micropapilar de CU es de interés para el urólogo y para el patólogo por varias razones: 1) el tumor infiltra la capa muscular propia en el momento del diagnóstico (72\% de los casos), por lo que el urólogo tiene que plantearse una nueva biopsia en profundidad cuando no se evidencie muscular propia en la resección inicial; 2) puede crecer por debajo de una mucosa normal ${ }^{28,30}$, lo que explicaría la dificultad de detectarlos tempranamente mediante cistoscopia o citología urinaria y la escasa eficacia de los tratamientos quimioterápicos intravesicales ${ }^{32}$ y 3 ) el valorar el posible origen vesical en una metástasis ganglionar cuando de observe un patrón micropapilar.

La variedad micropapilar de CU se asocia a CU convencional infiltrante de alto grado (56\%), a carcinoma urotelial in situ (60\%) y con menor frecuencia a adenocarcinoma ${ }^{26,27,29-31}$. En algunas series, incluso cuando el patrón micropapilar de $\mathrm{CU}$ es focal se asocia a mal pronóstico con supervivencias del $25 \%$ a los 5 años ${ }^{27}$. Sin embargo, otros autores encuentran una clara relación entre el porcentaje de carcinoma micropapilar, el estadio tumoral y el comportamiento clínico. En 


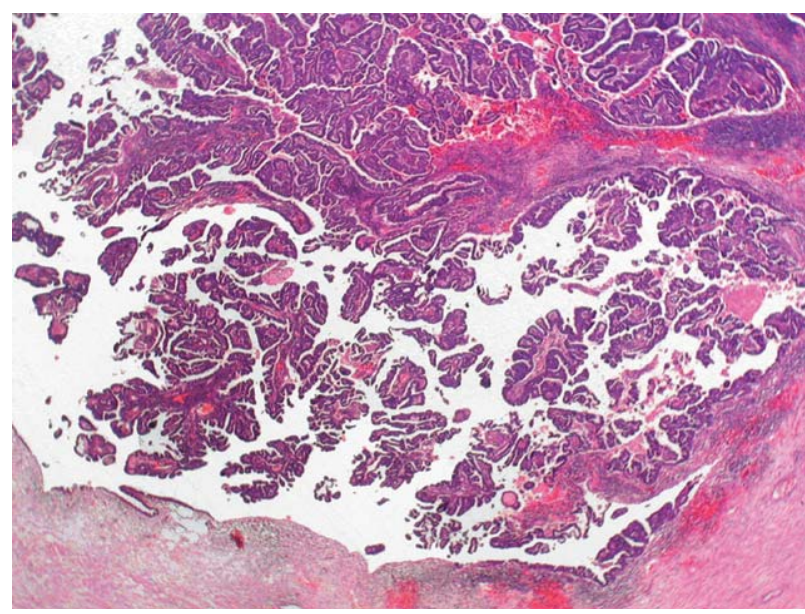

FIGURA 5a. Carcinoma urotelial con crecimiento de micropapilas en superficie.

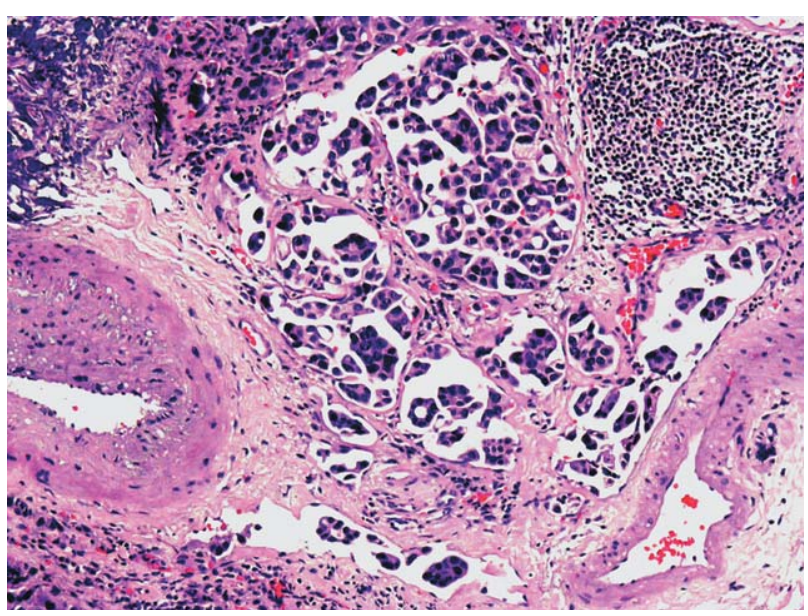

FIGURA 5b. Carcinoma urotelial micropapilar infiltrante.

la serie de Samaratunga ${ }^{30}$ todos los casos con componente micropapilar mayor 50\% y también la mayoría de los casos con 10-50\% debutaron en estadios avanzados. Por el contrario, el $85 \%$ de los casos con componente micropapilar menor del $10 \%$ tenían un estadio bajo al diagnóstico. Por lo tanto, es de interés que en los informes anatomopatológicos quede reflejado el porcentaje de componente micropapilar encontrado. En nuestra experiencia (24 casos), el $75 \%$ de las mujeres presentaron al diagnóstico componente micropapilar en el $100 \%$ del tumor que infiltraba extensamente todo el espesor de la pared vesical.

En la mayoría de las series publicadas el CU micropapilar se diagnostica en estadio pT2 o superior $^{26,27,29-31}$, lo que sugiere que la aproximación terapéutica de este tipo de tumor debe ser diferente al del CU convencional. Solamente una serie $^{32}$ evaluó retrospectivamente casos de CU micropapilar que se diagnosticaron en estadios pTa y pT1 valorando su comportamiento según los diferentes tratamientos recibidos. Los autores de este estudio recomiendan cistectomía radical debido a que en el $67 \%$ de los pacientes que recibieron quimioterapia intravesical se demostró progresión de la enfermedad (pT2 o mayor) y en el $22 \%$ metástasis en un tiempo medio de 8 meses después del tratamiento. Probablemente este mal pronóstico pueda estar condicionado por el infradiagnóstico clínico en la RTU diagnóstica ${ }^{32}$.

\section{CARCINOMA UROTELIAL CON ESTROMA LINFOIDE (CARCINOMA TIPO LINFOEPITELIOMA)}

$\mathrm{El}$ carcinoma tipo linfoepitelioma (CATLE) es un carcinoma indiferenciado primario de vejiga, que se acompaña de un denso infiltrado inflamatorio linfocitario, similar al linfoepitelioma de la nasofaringe.

El CATLE fue descrito por primera vez en la vejiga por Zukerberg et al. ${ }^{42}$ dentro de una revisión de carcinomas que pueden plantear problemas diagnósticos con linfomas y/o con plasmocitomas. El patrón histológico típico del CATLE se caracteriza por sábanas, cordones o trabéculas de células indiferenciadas entremezcladas con densos infiltrados de linfocitos maduros, acompañados de células plasmáticas, histiocitos y leucocitos polimorfonucleares neutrófilos y eosinófilos (Fig. 6). Las células tumorales son grandes

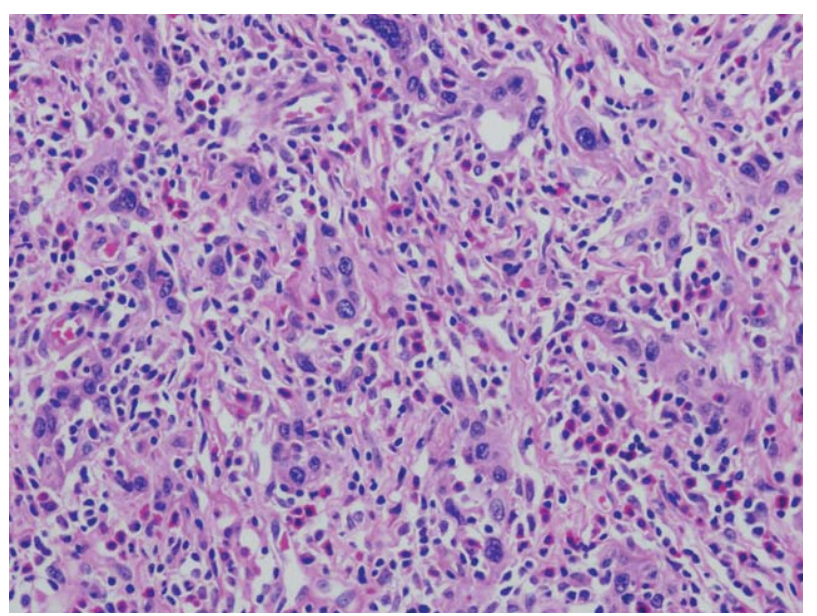

FIGURA 6. Carcinoma urotelial tipo linfoepitelioma con células epiteliales de aspecto sincitial con abundante infiltrado de linfocitos y eosinófilos. 
con citoplasmas mal definidos con núcleos vesiculosos con nucleolos prominentes y elevado indice mitótico ${ }^{42-48}$, siendo frecuentes los focos de necrosis ${ }^{42,48}$.

Según Amin et al. ${ }^{43}$, estos carcinomas pueden ser histológicamente puros (100\%), o estar asociados con carcinoma "in situ" y /o con un CU convencional superficial o infiltrante, a veces con diferenciación escamosa o glandular, siendo clasificados como CATLE predominante (mayor del $50 \%$ del tumor) y CATLE focal (menor del 50\%).

Con las técnicas de inmunohistoquímica, las células epiteliales tumorales son positivas con el antígeno epitelial de membrana (EMA) y con múltiples citoqueratinas ( AE1/AE3, CK7, CK8 CAM 5.2) (Fig. 7), pero la CK20 y las moléculas de adhesión CD44v6 sólo se expresan focalmente ${ }^{46}$. El componente linfoide está formado fundamentalmente por linfocitos T (CD3+), entremezclados con linfocitos B (CD20+) (Fig. 8), células dendríticas e histiocitos. No se ha demostrado relación entre el virus de Epstein-Barr y los CATLE de vejiga ${ }^{44-48}$.

La revisión de los casos descritos demuestra que son tumores infrecuentes, cuya incidencia representa el $0,4 \%-1,3 \%^{44,46}$ de todos los carcinomas de vejiga. La edad media de los pacientes es de 69 años (rango: 52-82 años), el 69\% son hombres, y el síntoma inicial más constante es la hematuria ${ }^{43-48}$. En la cistoscopia, los tumores suelen ser pequeños, solitarios y localizados en la pared libre de la vejiga ${ }^{44,45,47,48}$ (Fig. 9). En el 90\% de los casos, el tumor es infiltrante en el momento del diagnóstico ${ }^{42-48}$ y este dato debe de alertar al clínico, pues ante un diagnóstico histológico de CATLE, cuando la infiltración no es visible en la resección transuretral (RTU) diagnóstica, se tiene que considerar la posibilidad de una posterior evaluación quirúrgica.

Además, se ha visto que las formas histológicas puras y predominantes del CATLE, tienen mejor pronóstico que los carcinomas uroteliales infiltrantes, indiferenciados o pobremente diferenciados. Sin embargo, cuando el CATLE es focal, la evolución es similar al CU convencional de igual grado y estadio $^{43,44,46}$

Los resultados publicados parecen confirmar que los CATLE puros y predominantes son sensibles al tratamiento sistémico con quimioterapia y/o radioterapia ${ }^{42-47}$. Sin embargo, el número de

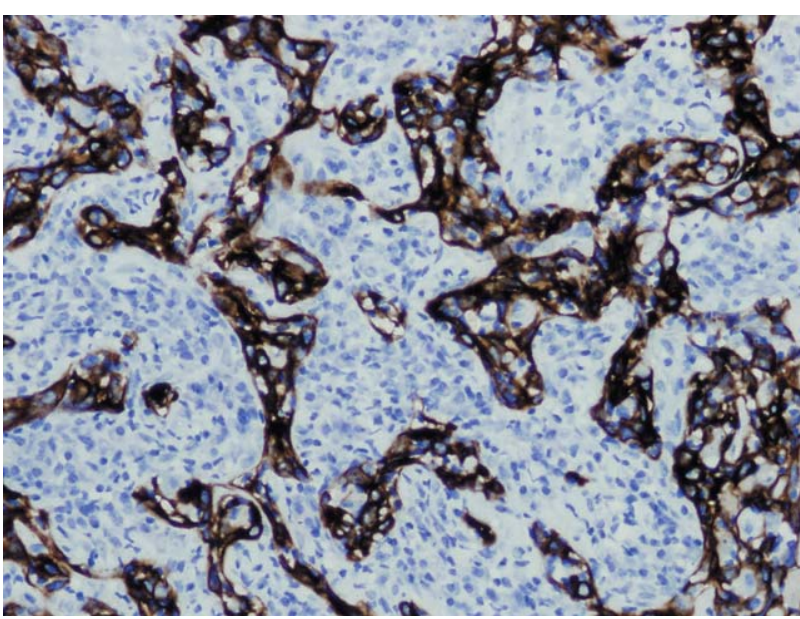

FIGURA 7. Carcinoma urotelial tipo linfoepitelioma con intensa inmunoexpresión de citoqueratina 7 en las células epiteliales.

casos descritos es pequeño y no hay protocolos terapéuticos establecidos. Posiblemente la RTU profunda, así como la cistectomía parcial, asociada a quimioterapia coadyuvante, podría evitar la cistectomía radical en estos tumores.

El CATLE puro debe distinguirse del linfoma. Los linfomas de la vejiga son tumores excepcionales, y casi siempre secundarios a linfomas de otra localización. En ocasiones, las células epiteliales malignas son dificiles de identificar en el seno del denso infiltrado linfoide, y la lesión puede ser interpretada como un proceso inflamatorio reactivo. En las biopsias endovesicales, no es infrecuente ver infiltrados inflamatorios crónicos asociados o no a un CU convencional, pero es

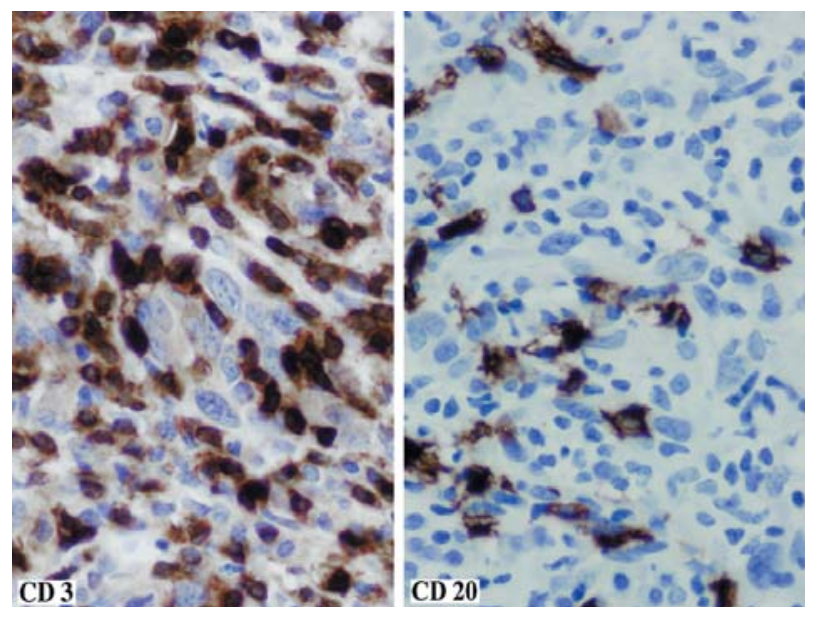

FIGURA 8. Carcinoma urotelial tipo linfoepitelioma. En el infiltrado linfoide predominan linfocitos T CD3 positivos. 


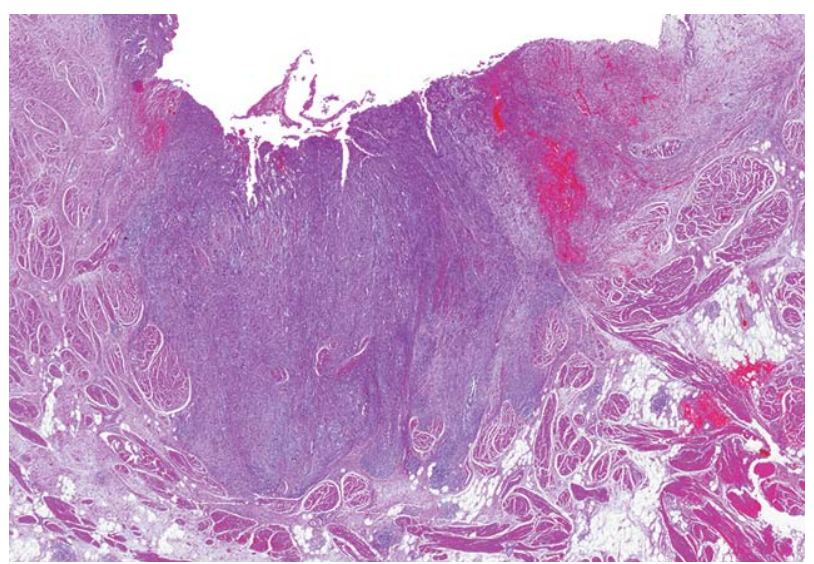

FIGURA 9. Carcinoma urotelial tipo linfoepitelioma. Nódulo único densamente celular, homogéneo que alcanza la muscular propia de la vejiga.

excepcional que la respuesta inflamatoria sea extensa y dominante. En estos casos, hay que sospechar la posibilidad de que exista un carcinoma indiferenciado asociado, sobre todo si la inflamación se extiende a la pared muscular. El estudio de las biopsias problemáticas con técnicas de inmunohistoquímica, con marcadores frente a queratinas (CK7, CK20, AE1,3) y con marcadores de células linfoides, son imprescindibles para resolver estos problemas diagnósticos.

Además, los carcinomas uroteliales convencionales pobremente diferenciados, que se acompañan de una importante respuesta inflamatoria, deben de ser diferenciados de los carcinomas tipo linfoepitelioma. Para el diagnostico de CATLE, las células epiteliales tumorales tienen que mostrar el característico patrón de crecimiento sincitial, con núcleos vesiculosos con nucleolos prominentes.

Por tanto, los datos clínico-evolutivos y terapéuticos apoyan que es importante reconocer el CATLE en su forma pura y predominante, como un peculiar patrón histológico de $\mathrm{CU}$, que ante el mismo grado y estadio tienen un pronóstico más favorable que el CU convencional, posiblemente debido al intenso infiltrado inflamatorio formado por abundantes linfocitos $\mathrm{T}$, expresión de una gran respuesta inmune frente a las células epiteliales atípicas, que expresan los marcadores inmunohistoquímicos del CU.

\section{CARCINOMA PLASMOCITOIDE}

Dentro de las variedades histológicas del CU, los carcinomas con hábito plasmocitoide, son muy infrecuentes, con sólo 8 casos descritos en la literatura ${ }^{49-56}$. Esta variedad histológica fue descrita casi simultáneamente por $\operatorname{Sahin}^{49}$ y Zukerberg ${ }^{50}$ en 1991 y en los dos trabajos se destaca el fenotipo plasmocitoide de las células neoplásicas, lo que justifica que estos tumores simulen un mieloma o un linfoma inmunoblástico con diferenciación plasmocitoide ${ }^{49,50}$. Histológicamente el tumor está formado por una proliferación difusa monomorfa de células poco cohesivas, que infiltran la lámina propia y la muscular propia de la vejiga. Las células son de mediano tamaño con citoplasmas eosinófilos o pálidos con núcleos excéntricos con cromatina homogénea, sin nucleolos evidentes. Las células infiltran la lámina propia y la pared muscular como células aisladas o en pequeños cordones acompañadas de un estroma mixoide o fibro-mixoide, escasamente celular (Fig. 10). Los tumores son infiltrantes en el momento del diagnóstico, y en ocasiones presentan extensión extravesical ${ }^{50,54}$. En dos casos, los pacientes presentaron múltiples lesiones osteolíticas, y en el caso descrito por Sahin et $a .^{49}$, fue la patología ósea, la primera manifestación clínica.

En cuatro de los casos publicados, el CU plasmocitoide estaba asociado con un CU "in situ" 53 , un CU convencional ${ }^{50,51}$ o un $\mathrm{CU}$ con patrón micropapilar ${ }^{55}$. En los tres pacientes estudiados por nosotros, en la cistectomía, el tumor infiltrante presentaba un patrón exclusivo de carcinoma plasmocitoide, estadio pT3 en dos casos y pT2 en un caso. En todos nuestros casos, la pieza quirúrgica o las RTU previas presentaban además un CU pobremente diferenciado. Esta asociación reafirma la naturaleza urotelial del carcinoma plasmocitoide.

Cuando el carcinoma plasmocitoide no tiene asociado un $\mathrm{CU}$, este tipo tumoral plantea varios problemas diagnósticos. En primer lugar, el patrón de crecimiento difuso, homogéneo, con células poco cohesivas, con núcleo excéntrico y citoplasma eosinófilo puede sugerir la infiltración vesical por un plasmocitoma. La positividad de las células tumorales con antisueros frente a las queratinas CK7 y CK20 (Fig.11a) y la negatividad con los marcadores de linfocitos y de células plasmáticas, apoyan la naturaleza epitelial del tumor. Si la muestra es pequeña y 


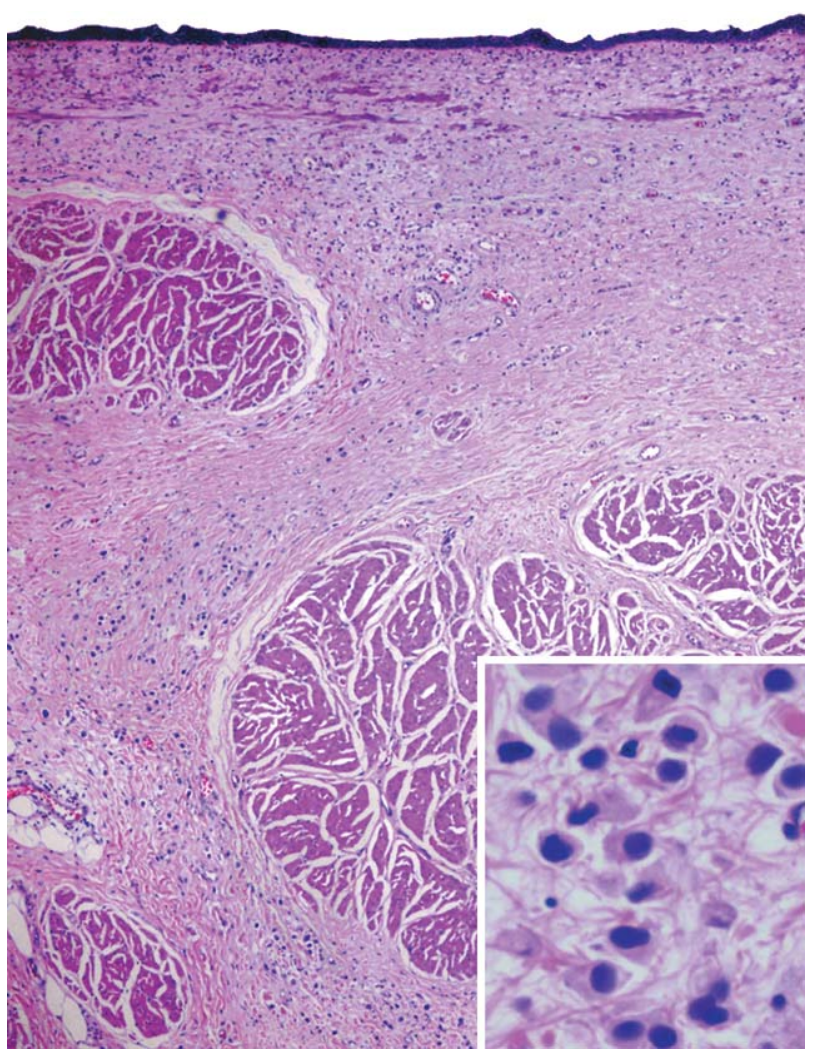

FIGURA 10. Carcinoma urotelial plasmocitoide. Pared vesical con intenso edema de la lámina propia e interfascicular con infiltración por células pequeñas no cohesivas con citoplasmas eosinófilos y núcleos homogéneos (Inset).

superficial, el tumor puede no ser sospechado, y la biopsia es interpretada como una lesión inflamatoria, sobre todo en aquellos casos en los que solo hay células tumorales plasmocitoides aisladas, acompañadas de intenso fibroedema. Valorar las características del núcleo de las células plasmocitoides, y conocer que este patrón histológico puede asociarse con edema de la lámina propia, son datos que pueden orientarnos sobre la posible naturaleza maligna de la lesión.

Hay muy pocos casos publicados de carcinomas plasmocitoides, pero en algunos se han descrito células tumorales con zonas claras paranucleares ${ }^{51}$, células con grandes vacuolas citoplasmáticas, simulando lipoblastos ${ }^{53}$ y células con morfología en "anillo de sello"54. Estos hallazgos, junto al patrón de crecimiento difuso del carcinoma plasmocitoide, puede plantear el diagnóstico diferencial con un adenocarcinoma con células en "anillo de sello" puro de vejiga, sobre todo con las formas más indiferenciadas, en las que las células tumorales tienen escasa o nula producción de mucina intracitoplasmática. Probablemente no sea trascendente distinguir si algunos carcinomas plasmocitoides son un adenocarcinoma con células en "anillo de sello", indiferenciadas o un CU indiferenciado, ya que esta distinción no va a tener implicaciones terapéuticas y el pronóstico va a estar condicionado por el estadio tumoral al diagnóstico. Sin embargo, si es importante conocer que algunos carcinomas vesicales pueden presentar un habito plasmocitoide, para no confundirlos con la infiltración vesical por un linfoma o un plasmocitoma. El patrón infiltrativo del carcinoma plasmocitoide de vejiga, con células aisladas poco cohesivas y en pequeños cordones, es muy similar al carcinoma lobulillar de mama, y al igual que ocurre en este tumor, la capacidad infiltrativa y metastatizante del carcinoma plasmocitoide de vejiga, puede estar relacionada con la ausencia de cadherina $\mathrm{E}^{56}$, hecho que hemos constatado en nuestros tres casos de carcinoma plasmocitoide de vejiga (Fig. 11b).

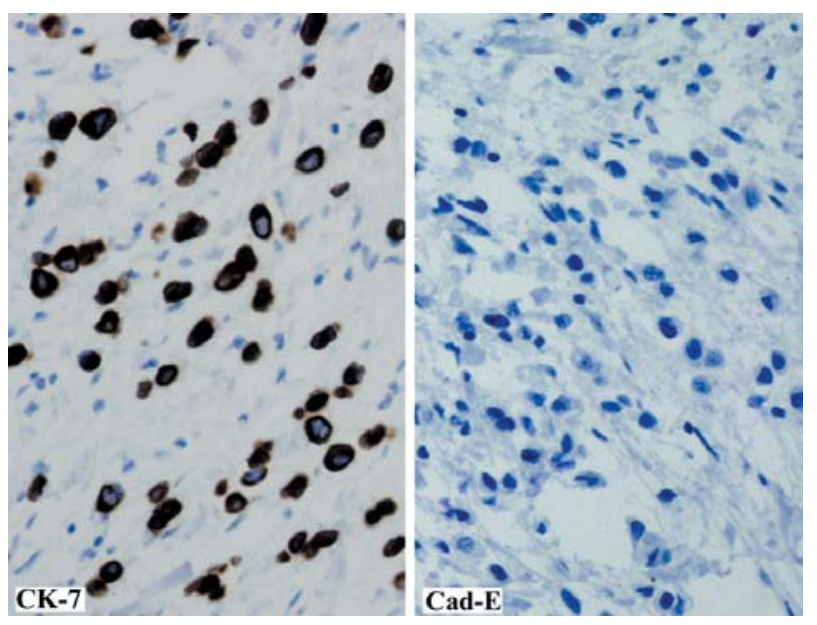

FIGURA 11. Carcinoma urotelial plasmocitoide. Las células tumorales expresan positividad con la citoqueratina 7 (izquierda) y pérdida de expresión de cadherina $E$ (derecha).

\section{CARCINOMA SARCOMATOIDE/ CARCINOSARCOMA}

La última revisión de la $\mathrm{OMS}^{3}$ establece que el término variante sarcomatoide de $\mathrm{CU}$ (con o sin elementos heterólogos) debe ser usado para defi- 
nir a todos aquellos tumores malignos bifásicos con evidencia morfológica y/o inmunohistoquímica de diferenciación epitelial y mesenquimal, especificando en el diagnóstico la presencia o ausencia de elementos heterólogos. Sin embargo, en la literatura existe una considerable confusión y desacuerdo acerca de la nomenclatura e histogénesis de esta variante de CU. Las características fenotípicas que se han incluido en la categoría "carcinoma sarcomatoide" han variado a lo largo del tiempo. Así, en algunas series carcinosarcoma y carcinoma sarcomatoide se consideran entidades diferentes, reservando el término carcinosarcoma para aquellos casos en los que existían elementos mesenquimales heterólogos ${ }^{57-60}$. Sin embargo, otras series ${ }^{61,62}$ argumentan que los detalles histológicos que separan a ambos tipos de tumores no tienen significado clínico aparente y por ello consideran a ambos una misma entidad que agrupan bajo el término "carcinoma sarcomatoide". Esta es la tendencia en los últimos años tal como ha recogido la última publicación de la OMS.

Los datos epidemiológicos y la presentación clínica, tanto en edad como en sintomatología, no difieren significativamente del CU convencional. Los carcinomas sarcomatoides se caracterizan macroscópica y cistoscópicamente por ser grandes masas exofiticas polipoides, parcialmente necróticas $\mathrm{y}$ ulceradas que protruyen en la luz e infiltran la pared vesical (Fig. 12a). Microscópicamente, el componente epitelial que se encuentra con mayor frecuencia es el CU (80\%), describiéndose también carcinoma epidermoide, adenocarcinoma y carcinoma de células pequeñas. El componente mesenquimal más frecuentemente observado es una neoplasia fusocelular indifenciada de alto grado de malignidad (Fig. 12b). Cuando existen elementos heterólogos, el más frecuentemente observado es el osteosarcoma (37\%), seguido de condrosarcoma, (Fig. 13a) rabdomiosarcoma (Fig. 13b y c), sarcoma fusocelular indiferenciado, leiomiosarcoma, liposarcoma y angiosarco$\mathrm{ma}^{3,59,60}$. En un mismo tumor pueden encontrarse más de un componente epitelial (33\%) o más
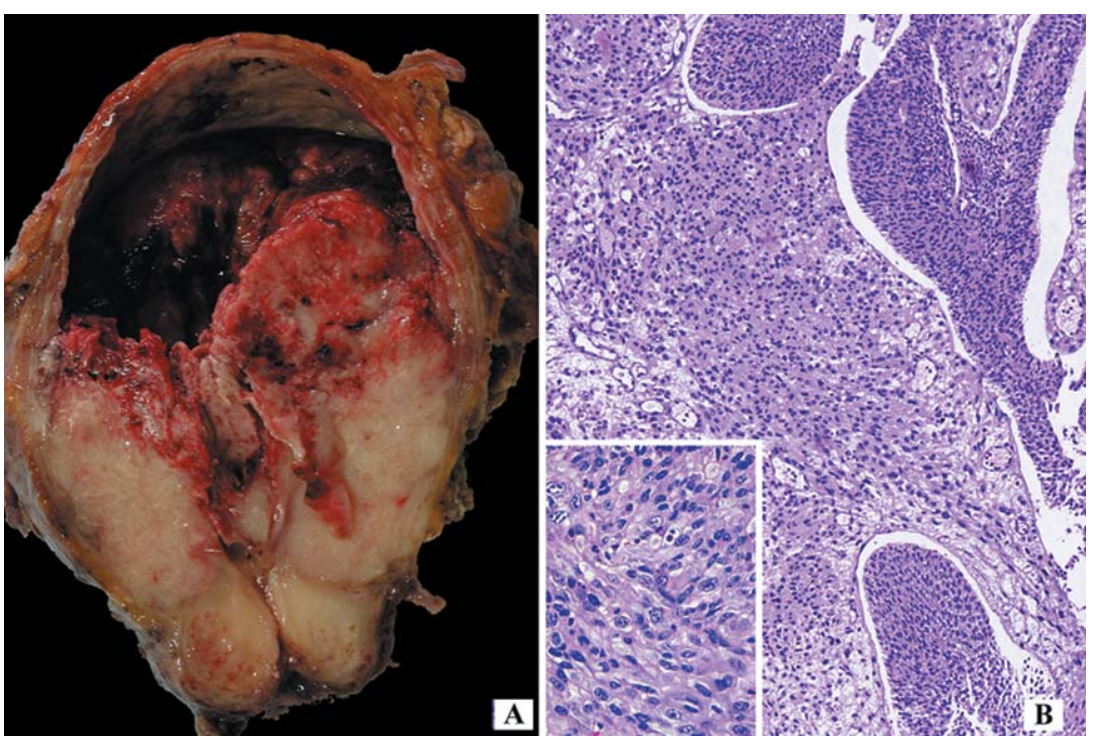

FIGURA 12. A) Carcinoma urotelial sarcomatoide. Gran masa tumoral exofitica, polipoide y ulcerada que ocupa gran parte de la luz vesical. El tumor presenta aspecto muco-condroide en amplias zonas. B) Componente epitelial y sarcomatoide con áreas de intensa atipia (inset).
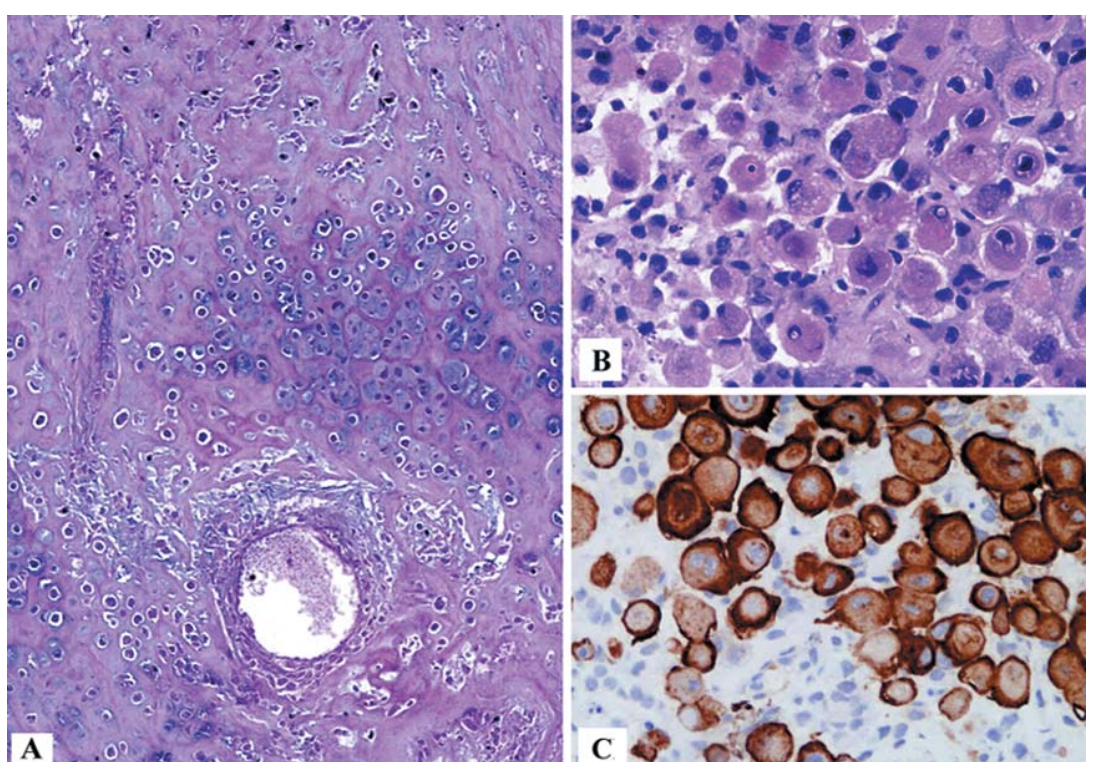

FIGURA 13. Carcinoma sarcomatoide con diferenciación heteróloga tipo condrosarcoma (A) y de rabdomiosarcoma (B) con expresión de desmina (C). 
de un componente mesenquimal (17\%) de los $\operatorname{casos}^{60}$. Estudios moleculares recientes apoyan el origen monoclonal de ambos componentes ${ }^{63}$. Un subtipo de carcinoma sarcomatoide es aquel que se acompaña de estroma mixoide abundante, por lo que puede plantear problemas de diagnóstico diferencial con los seudotumores inflamatorios ${ }^{64}$. El diagnóstico diferencial con lesiones mesenquimales benignas "pseudotumorales" de la vejiga, así como con CU con metaplasia ósea y cartilaginosa, se basa en la marcada atipia en el componente fusocelular del carcinoma sarcomatoide. Más del $80 \%$ de estos tumores son infiltrantes en el momento del diagnóstico ${ }^{59}$ y tienen un comportamiento clínico mucho más agresivo, independientemente del tratamiento, que el CU convencional. El $70 \%$ de los pacientes mueren por el tumor entre 1 y 48 meses después del diagnóstico (media 17 meses), aunque algunos pacientes han tenido supervivencias más prolongadas combinando cirugía y radioterapia. El estadio patológico parece ser el mejor factor pronóstico para predecir la supervivencia de los carcinomas sarcomatoides de vejiga ${ }^{58}$.

\section{REFERENCIAS}

1. Eble JN, Young RH. Carcinoma of the urinary bladder: a review of its diverse morphology. Semin Diagn Pathol. 1997;14(2):98-108.

2. Reuter VE. The urothelial tract: renal pelvis, ureter urinary bladder and urethra. In: Stenberg's Diagnostic Surgical Pathology. Mills SE, Carter D, Greenson JK, Oberman HA, Reuter RE, Stoler MH (Eds). Lippincott Williams \& Wilkins 2004; pp: 2035-2081.

3. Lopez- Beltran A, Sauter G, Gasser T, Hartmann A, Schmitz-Dräger, Helpap B. Infiltranting urothelial carcinoma; WHO classification of noninvasive papillary urothelial tumors. En World Health Organization classification of tumors. Pathology and genetics of tumors of the urinary system and male genital organs. Eble JN, Epstein JI, Sesterhenn I (Eds): Lyon, IARCC 2004.

\section{Carcinoma Urotelial Variedad en Nidos y/o Tubular}

4. Stern JB. Unusual benign bladder tumor of Brunn nest origin. Urology. 1979;14(3):288-289.

5. Talbert ML, Young RH. Carcinomas of the urinary bladder with deceptively benign-appearing foci. A report of three cases. Am J Surg Pathol. 1989;13(3):374-381.

6. Murphy WN, Deana DG. The nested variant of transitional cell carcinoma: a neoplasm resembling proliferation of Brunn`s nests. Mod Pathol. 1992;5(3):240-243.

7. Drew PA, Furman J, Civantos F, Murphy WN. The nested variant of transitional cell carcinoma: an aggressive neoplasm with innocuous histology. Mod Pathol. 1996;9(10): 989-994.
8. Young RH, Oliva E. Transitional cell carcinomas of the urinary bladder that may be underdiagnosed. A report of four invasive cases exemplifying the homology between neoplastic and nonneoplastic transitional cell lesions. Am J Surg Pathol. 1996;20(12):1448-1454.

9. Eble JN, Young RH. Carcinomas of the urinary bladder: A review of its diverse morphology. Sem Diagn Pathol. 1997; 14(2):98-108.

10. Badoual C, Bergemer-Fouquet AM, Renjard L, Lefrancq T, Lesourd B, Belhiba $\mathrm{H}$ et al: An unusual variant of urothelial carcinoma: the "nested variant of urothelial carcinoma" report of two cases. Ann Pathol. 1999;19(2):119-123.

11. Ozdemir BH, Ozdemir OG, Sertcelik A. The nested variant of the transitional cell bladder carcinoma: a case report and review of the literature. Int Urol Nephrol. 2000;32(2):257-258.

12. Holmang S, Johansson SL. The nested variant of transitional cell carcinoma: a rare neoplasm with poor prognosis. Scand J Urol Nephrol. 2001;35(2):102-105.

13. Tatsura H, Ogawa K, Sakata T, Okamura T. A nested variant of transitional cell carcinoma of the urinary bladder: a case report. Jap J Clin Oncol. 2001;31(6):287-289.

14. Liedberg F, Chebil G, Davidsson T, Gadaleanu V, Grabe M, Mansson W. The nested variant of urothelial carcinoma: a rare but important bladder neoplasm with aggressive behavior. Three case reports and a review of the literature. Urol Oncol. 2003;21(1):7-9.

15. Xiao GQ, Savage SJ, Gribetz ME, Burstein DE, Miller LK, Unger PD. The nested variant of urothelial carcinoma: clinicopathology of 2 cases. Arch Pathol Lab Med. 2003;127 (8):333-336.

16. Lin O, Cardillo M, Dalbagni G, Linkov I, Hutchinson B, Reuter VE. Nested variant of urothelial carcinoma: a clinicopathological and immunohistochemical study of 12 cases. Mod Pathol. 2003;16(12):1289-1298.

17. Volmar KE, Chan TY, De Marzo AM, Epstein JI. Florid von Brunn nests mimicking urothelial carcinoma: a morphologic and immunohistochemical comparison to the nested variant of urothelial carcinoma. Am J Surg Pathol. 2003; 27(9): 1243-1252.

18. Berthier A,Gobet F Bugel H, Roquet L, Pfister C. Nested variant urothelial carcinoma: diagnostic difficulties. Prog Urol. 2005; 15(4):729-732.

19. OOi SM, Vivian J, Sinniah R, Troon S. Nested variant of urothelial carcinoma: a rare presentation. Urology. 2006; $67(4): 845$.

\section{Carcinoma Urotelial Microquístico}

20. Young RH, Zukerberg LR. Microcystic transitional cell carcinomas of the urinary bladder. A report of four cases. Am J Clin Pathol.1991;96(5):635-639.

21. Leroy X, Leteurtre E, De La Taille A, Augusto D, Biserte J, Gosselin B. Microcystic transitional cell carcinoma. A report of 2 cases arising in the renal pelvis. Arch Pathol Lab Med. 2002;126(7):859-861.

22. Radopoulos D, Kalivas K, Kotakidou R, Panagiotopoulou K, Katsikas V, Papathanasiou M. Case report: Microcystic transitional cell carcinoma of the urinary bladder. Int Urol and Nephrol. 2005;37(2):291-293.

23. Paz A, Rath-Wolfson D, Lask D, Koren R, Manes A, Mukamel E, et al. The clinical and histological features of transitional cell carcinoma with microcysts: analysis of 12 cases. Br J Urol. 1997;79(5):722-725. 


\section{Carcinoma Urotelial Micropapilar}

24. Nassar H. Carcinomas with micropapillary morphology: clinical significance and current concepts. Adv Anat Pathol. 2004;11(6):297-303.

25. Nassar H, Pansare V, Zhang H, Che M, Sakr W, Ali-Fehmi $\mathrm{R}$, Grignon D, et al. Pathogenesis of invasive micropapillary carcinoma: role of MUC1 glycoprotein. Mod Pathol. 2004;17(9):1045-1050.

26. Amin MB, Ro JY, el-Sharkawy T, Lee KM, Troncoso P, Silva EG, et al. Micropapillary variant of transitional cell carcinoma of the urinary bladder. Histologic pattern resembling ovarian papillary serous carcinoma. Am J Surg Pathol. 1994;18(26): 1224-1232.

27. Johansson SL, Borghede G, Holmang S. Micropapillary bladder carcinoma: a clinicopathological study of 20 cases. J Urol. 1999;161(6):1798-1802.

28. Maranchie JK, Bouyounes BT, Zhang PL, O'Donnell MA, Summerhayes IC, DeWolf WC. Clinical and pathological characteristics of micropapillary transitional cell carcinoma: a highly aggressive variant. J Urol. 2000;163(3):748-751.

29. Ylagan LR, Humphrey PA. Micropapillary variant of transitional cell carcinoma of the urinary bladder: a report of three cases with cytologic diagnosis in urine specimens. Acta Cytol. 2001;45(4):599-604.

30. Samaratunga H, Khoo K. Micropapillary variant of urothelial carcinoma of the urinary bladder; a clinicopathological and immunohistochemical study. Histopathology. 2004; 45(1):55-64.

31. Alvarado-Cabrero I, Sierra-Santiesteban FI, MantillaMorales A, Hernandez-Hernandez DM. Micropapillary carcinoma of the urothelial tract. A clinicopathologic study of 38 cases. Ann Diagn Pathol. 2005;9(1):1-5.

32. Kamat AM, Gee JR, Dinney CP, Grossman HB, Swanson DA, Millikan RE, et al. The case for early cystectomy in the treatment of nonmuscle invasive micropapillary bladder carcinoma. J Urol. 2006;175(3 Pt 1):881-885.

33. Lopez JI, Elorriaga K, Imaz I, Bilbao FJ. Micropapillary transitional cell carcinoma of the urinary bladder. Histopathology. 1999;34(6):561-562.

34. Vang R, Abrams J. A micropapillary variant of transitional cell carcinoma arising in the ureter. Arch Pathol Lab Med. 2000;124(9): 1347-1348.

35. Oh YL, Kim KR. Micropapillary variant of transitional cell carcinoma of the ureter. Pathol Int. 2000;50(1):52-56.

36. Santos-Briz A, Antunez P, Serrano A, Ballestin C, Usera G. Carcinoma micropapilar de vejiga: presentación de un caso y revisión de la literatura. Rev Esp Patol. 2001;34: 153-156.

37. Vera J, Marigil M, Garcia MD, Abascal M, Sanz JI Micropapillary bladder carcinoma. Virchows Arch. 2002; 441(4):412-413.

38. Sugino Y, Negoro H, Iwamura H, Moroi S, Oka H, Kawakita $\mathrm{M}$, et al. Micropapillary variant of transitional cell carcinoma of the bladder. Int J Urol. 2004;11(9):792-794.

39. Chan GS, Ng WK, Nicholls JM, Chan KW. Pathologic quiz case: acute renal failure secondary to an uncommon urinary bladder tumor. Micropapillary transitional cell carcinoma of urinary bladder. Arch Pathol Lab Med. 2005;129 (2):e53-54.

40. Nishizawa K, Kobayashi T, Mitsumori K, Ide Y, Watanabe J, Ogura K. Micropapillary bladder cancer. Int J Urol 2005; 12(5):506-508.
41. Ripa Saldias L, Guarch Troyas R, Hualde Alfaro A, De Pablo Cardenas A, Pinos Paul M, Santiago Gonzalez de Garibay A. Micropapillary carcinoma of the bladder: case report and review of the literature. Actas Urol Esp. 2005; 29(4):408-413.

\section{Carcinoma Urotelial con Estroma Linfoide (Carcinoma tipo} Linfoepitelioma)

42. Zukerberg LR, Harris NL, Young RH. Carcinomas of the urinary bladder simulating malignant lymphoma. A report of five cases. Am J Surg Pathol. 1991;15(6):569-576.

43. Amin MB, Ro JY, Lee KM, Ordoñez NG, Dinney CP, Gulley ML et al. Lymphoepithelioma-like carcinoma of the urinary bladder. Am J Surg Pathol. 1994;18(5):466-473.

44. Holmang S, Borghede G, Johansson S. Bladder carcinoma with lymphoepitelioma like differentiation: a report of 9 cases. J Urol. 1998;159(3):779-782.

45. Constantinides C, Giannopoulos A, Kyriakou G, Androulaki A, Ioannou M, Dimopoulos M, et al. Lymphoepithelioma-like carcinoma of the bladder. BJU 2001;87(1):121122.

46. Lopez-Beltran A, Luque RJ, Vicioso L, Anglada F, Requena MJ, Quintero A. et al. Lymphoepithelioma-like carcinoma of the urinary bladder: a clinicopathologic study of 13 cases. Virchows Arch. 2001;438(6):552-557.

47. Porcaro AB, Gilioli E, Migliorini F, Antoniolli SZ, Iannucci A, Comunale L. Primary lymphoepithelioma-like carcinoma of the urinary bladder: Report of one case with review and update of the literature after a pooled analysis of 43 patients. Int Urol Nephrol. 2003;35(1):99-106.

48. Guresci S, Doganay L, Altaner S, Atakan HI, Kutlu K. Lymphoepithelioma-like carcinoma of the urinary bladder: a case report and discussion of differential diagnosis. Int Urol Nephrol. 2005;37(1):65-68.

\section{Carcinoma Plasmocitoide}

49. Sahin AA, Myhre M, Ro JY, Sheige N, Ayala AG. Plasmacytoid transitional cell carcinoma. Report of a case and review of the literature. Acta Cytol. 1991;35(3):277280.

50. Zukerberg LR, Harris NL, Young RH. Carcinomas of the urinary bladder simulating malignant lymphoma. A report of five cases. Am J Surg Pathol. 1991;15(6):569-576.

51. Toledo G, Gracia A, Panizo A, Pardo-Mindan J. Plasmacytoid transitional cell carcinoma of the urinary bladder: A case report and review of the literature. Rev Esp Patol. 2001;34:65-68.

52. Zhang XM, Elhosseiny A, Melamed MR. Plasmacytoid urothelial carcinoma of the bladder. A case report and the first description of urinary cytology. Acta Cytol. 2002;46(2): 412-416.

53. Soylu A, Aydin NE, Yilmaz U, Kutlu R, Gunes A. Urothelial carcinoma featuring lipid cell and plasmacytoid morfology with poor prognostic outcome. Urology. 2005;65(4):797.

54. Mitsogiannis IC, Ioannou MG, Sinani CD, Melekos MD. Plasmacytoid transitional cell carcinoma of the urinary bladder. Urology. 2005;66(1): 194.

55. López JI, Etxezarraga MC, Ereño C, Unda M Bilbao FJ. Carcinoma urotelial mixto micropapilar y plasmocitoide de vejiga. Rev Esp Patol. 2005;38:121-124.

56. Baldwin L, Lee AHS, Al-Talib RK, Theaker JM. Transitional cell carcinoma of the bladder mimicking lobular carcinoma of the breast variant of urothelial carcinoma. Histopathology. 2005;46(1):50-56. 


\section{Carcinoma Sarcomatoide/Carcinosarcoma}

57. Young RH. Carcinosarcoma of the urinary bladder. Cancer. 1987;59(7):1333-1339.

58. Lopez-Beltran A, Pacelli A, Rothenberg HJ, Wollan PC, Zincke H, Blute ML, et al. Carcinosarcoma and sarcomatoid carcinoma of the bladder: clinicopathological study of 41 cases. J Urol. 1998;159(5):1497-1503.

59. Perret L, Chaubert P, Hessler D, Guillou L. Primary heterologous carcinosarcoma (metaplastic carcinoma) of the urinary bladder: a clinicopathologic, immunohistochemical, and ultrastructural analysis of eight cases and a review of the literature. Cancer. 1998;82(8):1535-1549.

60. Baschinsky DY, Chen JH, Vadmal MS, Lucas JG, Bahnson RR, Niemann TH. Carcinosarcoma of the urinary bladderan aggressive tumor with diverse histogenesis. A clinicopathologic study of 4 cases and review of the literature. Arch Pathol Lab Med. 2000;12(8):1172-1188.

61. Reuter VE. Sarcomatoid lesions of the urogenital tract. Sem Diagn Pathol. 1993;10(2):188-201.
62. Serio G, Zampatti C, Ceppi M. Spindle and giant cell carcinoma of the urinary bladder: a clinicopathological light microscopic and immunohistochemical study. $\mathrm{Br} \mathrm{J}$ Urol. 1995;75(2):167-172.

63. Halachmi S, DeMarzo AM, Chow NH, Halachmi N, Smith $\mathrm{AE}$, Linn JF, et al. Genetic alterations in urinary bladder carcinosarcoma: evidence of a common clonal origin. Eur Urol. 2000;37(3):350-357.

64. Jones EC, Young RH. Myxoid and sclerosing sarcomatoid transitional cell carcinoma of the urinary bladder: a clinicopathologic and immunohistochemical study of 25 cases. Mod Pathol. 1997;10(9):908-1016.

Correspondencia autora: Dra. $\mathrm{M}^{\mathrm{a}} \mathrm{L}$. Picazo Departamento de Anatomía Patológica Hospital Universitario La Paz

Castellana, 261 - 28046 Madrid. Tel.: 917277049

E-mail autora: mlpicazol.hulp@salud.madrid.org

Información artículo: Original - Vejiga 